\title{
Effect of Aging on Tympanometric Findings in Indian Population
}

\author{
Sujeet Kumar Sinha ${ }^{1, \odot}$ Anuj Kumar Neupane $e^{1,2, \odot}$ \\ ${ }^{1}$ Department of Audiology, All India Institute of Speech and \\ Hearing, Mysore, Karnataka, India \\ ${ }^{2}$ School of Audiology and Speech Language Pathology, Bharati \\ Vidyapeeth (Deemed to be University), Pune, Maharashtra, India
}

\author{
Krithika Gururaj'
}

\begin{abstract}
Address for correspondence Anuj Kumar Neupane, MSc (Audiology), School of Audiology and Speech Language Pathology, Bharati Vidyapeeth (Deemed to be University), Pune - 411043, Maharashtra, India (e-mail: anujkneupane@gmail.com).
\end{abstract}

\begin{abstract}
Keywords

- presbycusis

- tympanometric width

- ear canal volume

- static admittance

Introduction Presbycusis, or age-related hearing loss being a cumulative effect of aging on hearing, is a progressive and irreversible bilateral symmetrical sensorineural loss. In older individuals, one of the most important structures which undergo changes due to aging is the middle ear. There is a dearth of studies in Indian population regarding the tympanometric characteristics in older individuals. Thus, this study was taken up with an objective to report the different tympanometric characteristics in elderly individuals (aged above 50 years) who reported with ear- or hearing-related complaints to the Department of Audiology, All India Institute of Speech and Hearing, Mysore, Karnataka, India.

Materials and Methods A register-based analysis was conducted where immittance tests were performed.

Results There was no effect of age on static admittance, ear canal volume, and tympanometric peak pressure values. Also, there was no gender effect on tympanometric findings.

Conclusion The result of the present study helps in understanding the different middle ear mechanisms in older adults.
\end{abstract}

\section{Introduction}

There are about 285 million individuals in India aged above 50 years as per the 2001 census. ${ }^{1}$ Older individuals constitute one of the most rapidly growing segments in our population. Aging is usually associated with an overall decline in cognitive processes, like memory and thinking, and sensory abilities like speech perception. The speech understanding difficulties expressed by older adults are likely to arise from numerous changes that take place due to aging. With advance in age, it is understood that numerous structural and functional changes take place from the external ear to the brain. ${ }^{2}$

Published online August 25, 2021
DOI https://doi.org/ $10.1055 / \mathrm{s}-0041-1731921$ ISSN 2581-9607
In older individuals, one of the most important structures which undergo changes due to aging is the middle ear. In the middle ear the changes which occur due to aging are changes in thickness, vascularity, elastin, collagen, and cellularity of tympanic membrane.,4 It has also been reported that tympanic membrane in the older population with no history of middle ear pathology is denser and less transparent with the decomposition of calcium molecules degrading the elastic nature of the membrane. ${ }^{5}$ There are also reports of reduction in elastic tissue of the ossicular joints-incudomalleal and incudostapedial joints, 6,7 the ossicles ossification, and atrophic changes in the middle ear ligaments and muscle

(C) 2021. Indian Society of Otology.

This is an open access article published by Thieme under the terms of the Creative Commons Attribution-NonDerivative-NonCommercial-License, permitting copying and reproduction so long as the original work is given appropriate credit. Contents may not be used for commercial purposes, or adapted, remixed, transformed or built upon. (https://creativecommons.org/licenses/by-nc-nd/4.0/).

Thieme Medical and Scientific Publishers Pvt. Ltd. A-12, 2nd Floor Sector 2, Noida-201301 UP, India 
fibres, ${ }^{3,4}$ with increase in age. Considering these anatomical changes in the older population, one would expect changes in various tympanometric findings in older subjects.

There are equivocal findings in literature with regard to various tympanometric results in older subjects. For example, few authors have reported reduced acoustic admittance with increase in age, ${ }^{8-11}$ whereas, few others have reported no significant change in acoustic admittance with increase in age. ${ }^{12-15}$ Also the ear canal volume results in older subjects show conflicting results across the various studies. Several investigators have reported reduced ear canal function in older subjects. ${ }^{16-18}$ Others have reported no significant changes in ear canal volume in older participants. ${ }^{11,19}$ Similarly, there are equivocal findings with regard to tympanometric peak pressure in older participants. ${ }^{16-20}$

All these studies report tympanometric findings and middle ear characteristics in the western population. To our best of knowledge, there are no published reports concerned with tympanometric findings in the older Indian population. It is necessary to study the tympanometric findings in different population as it has been reported that the tympanometric findings may vary according to different races. Shahnaz et $a l,{ }^{21}$ reported that Chinese population have significantly lower static admittance, wider tympanometric width, more positive tympanometric peak pressure, and lower ear canal volume compared to Caucasian counterparts. Moreover, Beers et $\mathrm{al}^{22}$ reported that Chinese children were having lower energy reflectance values over the mid-frequency range compared to Caucasian counterparts. Another study by Wan and Wong, ${ }^{23}$ reported that there was significantly lower tympanometric peak pressure, wider tympanometric width, and more positive tympanometric peak pressure values in southern Chinese subjects compared to the other population. Thus, this study was taken up with an objective to report the different tympanometric characteristics (tympanometric peak pressure, static admittance, and ear canal volume) in the elderly individuals (aged above 50 years) who reported with the ear or hearing-related complaints to Department of Audiology, All India Institute of Speech and Hearing, Mysore between July 2013 and February 2014.

\section{Materials and Methods}

\section{Participants}

During this period of study, the tympanometric findings of 593 individuals with the age range 50 to 98 years were reviewed. Out of 593 individuals, 342 were females and 252 were male subjects. Further, the group was classified into five subgroups: Subgroup 1 consisted of 9 male subjects and 15 female subjects in the age range of 50 to 60 years; Subgroup 2 consisted of 139 male subjects and 206 female subjects in the age range of 61 to 70 years; Subgroup 3 consisted of 74 male subjects and 92 female subjects in the age range of 71 to 80 years; Subgroup 4 had 29 male subjects and 28 female subjects in the age range of 81 to 90 years; and Subgroup 5 had 1 male subject and 1 female subject in the age range of more than 90 years.

\section{Procedure}

A register-based, retrospective study was employed reviewing the clinical reports of cases who visited the Department of Audiology, All India Institute of Speech and Hearing (AIISH), Mysore, India in between July 2013 and February 2014, reporting the complaints related to ear and/or hearing loss. Various parameters relating to immittance such as type of tympanogram, tympanometric peak pressure, static admittance, ear canal volume were noted from the files. The audiological testing at the Department of Audiology was done by qualified audiologists.

\section{Results}

The mean and standard deviation for tympanometric peak pressure, static admittance, and ear canal volume for both genders were calculated and thus the summary data and statistics by age group, gender, and ear are represented in - Table 1 as well as -Figs. 1 and 2 ).

The data was subjected to the normality check. A Kolmogorov-Smirnov test was administered to check the normal distribution of data. Kolmogorov-Smirnov test revealed a non-normal distribution of the data $(p<0.05)$. Hence, a nonparametric test was done to analyze the data. To understand the significant differences in various tympanometric findings, Kruskal-Wallis test was done for findings within the male group. Within male group, Kruskal-Wallis test did not reveal a significant difference for tympanometry peak pressure, static admittance value, and ear canal volume for both ears $(p>0.05)$. Also, within female group, Kruskal-Wallis test was administered to determine a significant difference in immittance findings across the age groups. No significant difference for tympanometric peak pressure, static admittance value, and ear canal volume for both ears $(p>0.05)$ was found.

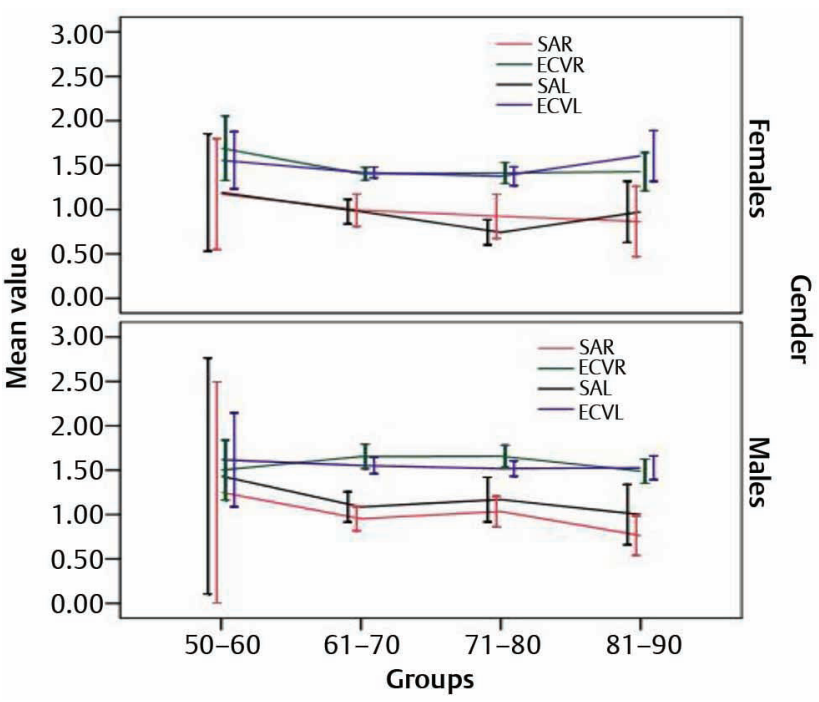

Fig. 1 Mean and standard deviation of static admittance and ear canal volume for right and left ear across gender for all age groups. ECVL, ear canal volume for left ear; ECVR, ear canal volume for right ear; SAL, static admittance for left ear; SAR, static admittance for right ear. 
Nonparametric Mann-Whitney test was done to compare

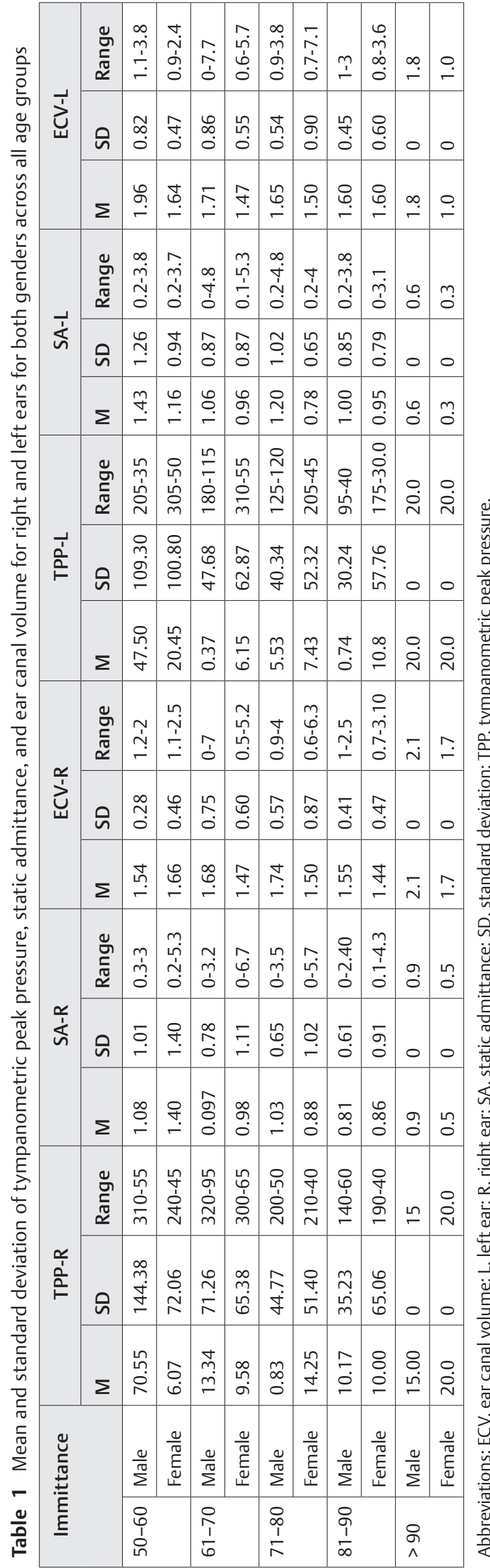

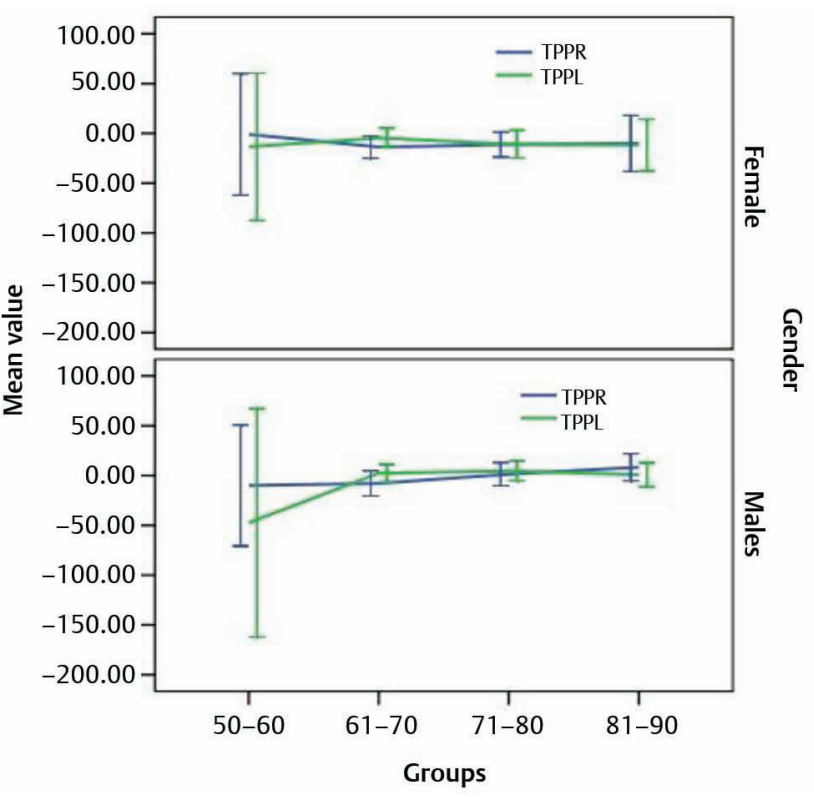

Fig. 2 Mean and standard deviation of tympanometric pressure for right and left ear across gender in all age groups. TPPL, tympanometric peak pressure for left ear; TPPR, tympanometric peak pressure for right ear.

the immittance findings across the two genders and results revealed that in the age group of 61 to 70 years, there was significant difference for ear canal volume for right ear $(Z=4.07$, $p<0.05)$ and for the left ear $(Z=3.67, p<0.05)$. In the age group of 71 to 80 years, immittance findings revealed significant differences of ear canal volume of the right ear $(Z=4.41$, $p<0.05)$, of the left ear $(Z=3.51, p<0.05)$, tympanometric peak pressure of the right ear $(Z=2.23, p<0.05)$, and static admittance of the right ear $(Z=3.18, p<0.05)$ and of the left ear $(Z=2.82, p<0.05)$. In the age group of 81 to 90 years, immittance findings revealed no significant difference for both ears $(p>0.05)$.

\section{Discussion}

In our present study, to understand the effect of age on middle ear characteristics, the tympanometric results have been analyzed in Indian older adults. In the present study, no significant difference in tympanometric peak pressure with age was found within the male or female group. The results of the present study are consistent with the previous studies. ${ }^{16,19,24,25}$ The middle ear pressure at $226 \mathrm{~Hz}$ probe tone correlates with tympanometric peak pressure because of a normal eustachian tube functioning. ${ }^{26}$ Hence, the variation in tympanometric peak pressure due to aging will also depend upon the functioning of the eustachian tube. There are studies to suggest that aging does not have a significant effect on eustachian tube functioning. ${ }^{19,24}$ Hence, the effect of aging was not observed for tympanometric peak pressure in the present study.

In the present study, we did not observe any age effect on the admittance values in both the male and female group. Previous studies have reported an equivocal finding regarding 
the effect of age on static admittance value, wherein few studies have reported a change in admittance values with an increase in age $\mathrm{e}^{9-11}$, whereas, other few have reported no change in static admittance values with an increase in age. ${ }^{15,19,25,27}$ The equivocal findings across the studies could be because of the different sample sizes taken for the studies. For example, Wiley et $\mathrm{al}^{16}$ studied tympanometric findings in 1,240 participants, Golding et $\mathrm{al}^{28}$ in 1,565 participants, and Uchida et $\mathrm{al}^{25}$ in 933 participants. In the present study, we had only 593 participants which were lesser compared to the previous studies; however, the results of the present study are consistent with some of the previous studies that there is no change in admittance value with age.

The statistical analysis has revealed no change in ear canal volume in the male and female group in our study. Similar results were obtained by Stenklev et $\mathrm{al}^{19}{ }^{19}$ wherein they also reported no changes in ear canal volume with respect to age in older adults. Although few studies indicate change in ear canal volume with increase in age, Wiley et al ${ }^{16}$ reported that there is a decrease in ear canal volume with advancing age, and the possible causes might be atrophic alterations of the walls of the canal, collapsed ear canal, and increased hair growth. ${ }^{17,18}$

The results of the present study revealed significant difference for ear canal volume from 61 to 80 years for both ears, whereas significant difference for tympanometric peak pressure and static admittance was found only for subjects in the age range of 71 to 80 years. Results for the other age groups were not significant. Thus, the results do not reveal any specific trend for gender difference. Previous studies have shown an equivocal effect of gender on various tympanometric findings. ${ }^{9,10,19,28,29}$ The results of the present study support some of the previous literature that there is no gender difference in various tympanometric findings.

\section{Conclusion}

In summary, results of the present study indicated no significant age effect on tympanometric peak pressure, static admittance, and ear canal volume in older adults. One of the initial research questions was concerned with the potential sensitivity of various tympanometric measures in demonstrating any stiffening or change in mass dominance of the middle ear transmission system with age. In this regard, findings of the present study revealed no unique middle ear aging effects on tympanometric peak pressure, static admittance, and ear canal volume. Also, there was no gender effect on abovementioned tympanometric findings. To conclude, there are no major changes in the tympanometric findings in older adults.

\section{Ethical Statement}

All participants were informed initially about the study in detail and a written consent form was obtained from all the participants. All the authors have equal contribution in data collection, data analysis, and manuscript preparation. The manuscript has not been submitted to any other journal.

\section{Funding}

None.

\section{Conflicts of Interest}

None.

\section{References}

1 Bose AB, Gangrade KD, Aging in India. New Delhi: Abhinav Publications; 2001

2 Willott JF. Aging and the Auditory System: Anatomy, Physiology and Psychophysics. San Diego: Singular Publishing Group; 1991

3 Covell WP. Histologic changes in the aging cochlea. J Gerontol 1952;7(2):173-177

4 Rosenwasser H. Otitic problems in the aged. Geriatrics 1964; 19:11-17

5 Ruah CB, Schachern PA, Zelterman D, Paparella MM, Yoon TH. Age-related morphologic changes in the human tympanic membrane. A light and electron microscopic study. Arch Otolaryngol Head Neck Surg 1991;117(6):627-634

6 Harty M. Elastic tissue in the middle-ear cavity. J Laryngol Otol 1953;67(12):723-729

7 Etholm B, Belal A Jr. Senile changes in the middle ear joints. Ann Otol Rhinol Laryngol 1974;83(1):49-54

8 Alberti PW, Kristensen R. The compliance of the middle ear: its accuracy in routine clinical practice. In: Rose D, Keating L, eds. Impedance Symposium. Rochester, MN: Mayo Clinic; 1972:159-167

9 Jerger J, Jerger S, Mauldin L. Studies in impedance audiometry. I. Normal and sensorineural ears. Arch Otolaryngol 1972; 96(6):513-523

10 Blood I, Greenberg HJ. Acoustic admittance of the ear in the geriatric person. J Am Audiol Soc 1977;2(5):185-187

11 Hall JW. Effects of age and sex on static compliance. Arch Otolaryngol 1979;105(3):153-156

12 Nerbonne MA, Bliss AT, Schow RL. Acoustic impedance values in the elderly. J Am Aud Soc 1978;4(2):57-59

13 Osterhammel D, Osterhammel P. Age and sex variations for the normal stapedial reflex thresholds and tympanometric compliance values. Scand Audiol 1979;8(3):153-158

14 Thompson DJ, Sills JA, Recke KS, Bui DM. Acoustic reflex growth in the aging adult. J Speech Hear Res 1980;23(2):405-418

15 Wilson RH. The effects of aging on the magnitude of the acoustic reflex. J Speech Hear Res 1981;24(3):406-414

16 Wiley TL, Cruickshanks KJ, Nondahl DM, Tweed TS, Klein R, Klein BE. Tympanometric measures in older adults. J Am Acad Audiol 1996;7(4):260-268

17 Hinchcliffe R. The anatomical locus of presbycusis. J Speech Hear Disord 1962;27(4):301-310

18 Chandler JR. Partial occlusion of the external auditory meatus: its effect upon air and bone conduction hearing acuity Laryngoscope 1964;74(1):22-54

19 StenklevNC,VikO,LaukliE. The agingear: an otomicroscopic and tympanometric study. Acta Otolaryngol 2004;124(1):69-76

20 Nondahl DM, Cruickshanks KJ, Wiley TL, Tweed TS, Dalton DS. Sixteen-year change in acoustic-admittance measures among older adults: data from a population-based study. J Speech Lang Hear Res 2013;56(6):1745-1750

21 Shahnaz N, Davies D. Standard and multifrequency tympanometric norms for Caucasian and Chinese young adults. Ear Hear 2006;27(1):75-90

22 Beers AN, Shahnaz N, Westerberg BD, Kozak FK. Wideband reflectance in normal Caucasian and Chinese school-aged children and in children with otitis media with effusion. Ear Hear 2010;31(2):221-233 
23 Wan IK, Wong LL. Tympanometric norms for Chinese young adults. Ear Hear 2002;23(5):416-421

24 Chermak GD, Moore MK. Eustachian tube function in the older adult. Ear Hear 1981;2(4):143-147

25 Uchida $\mathrm{Y}$, Nomura $\mathrm{H}$, Itoh A, et al. The effects of age on hearing and middle ear function. J Epidemiol 2000;10(suppl 1): S26-S32

26 Zemlin WR, Speech and Hearing Science: Anatomy and Physiology. Boston: Allyn \& Bacon; 2010
27 Holte L. Aging effects in multifrequency tympanometry. Ear Hear 1996;17(1):12-18

28 Golding M, Mitchell P, Cupples L. Risk markers for the graded severity of auditory processing abnormality in an older Australian population: the Blue Mountains Hearing Study. J Am Acad Audiol 2005;16(6):348-356

29 Liu XZ, Yan D. Ageing and hearing loss. J Pathol 2007;211(2): 188-197 